ДЛЯ КОРРЕСПОНДЕНЦИИ

Епифанцев Евгений Андреевич врач-хирург отделения хирургии ФГБУ ФНКЦ ФМБА России (Москва, Российская Федерация)

E-mail: epifantsev.e@gmail.com https://orcid.org/0000-0001-9768-

Ключевые слова: пластика пищевода, тонкокишечный трансплантат, аортомезентериальное шунтирование Evgeniy A. Epifantsev - Surgeon at Surgical Department, Federal

Scientific Medical Center of the FMBA of Russia (Moscow, Russian Federation) E-mail:epifantsev.e@gmail.com https://orcid.org/0000-0001-9768-

\section{Пластика пищевода}

\section{гиперваскуляризированным}

\section{тонкокишечным трансплантатом}

\section{в онкологической практике}

\author{
Ищенко Р.В. ${ }^{1}$, Епифанцев Е.А. ${ }^{2}$, Зотов А.С. ${ }^{2}$, Головинский С.В. ${ }^{2}$, Грицун В.Ю. ${ }^{2}$, \\ Смирнов A.B. ${ }^{2}$ \\ 1 Частное учреждение здравоохранения «Центральная клиническая больница “РЖД-Медицина"», 129128, \\ г. Москва, Российская Федерация \\ 2 Федеральное государственное бюджетное учреждение «Федеральный научно-клинический центр специализи- \\ рованных видов медицинской помощи и медицинских технологий Федерального медико-биологического агент- \\ ства», 115682, г. Москва, Российская Федерация
}

Авторы приводят собственное клиническое наблюдение успешного хирургического лечения пациента с первично-множественным синхронным раком легкого и желудка. Пластическая реконструкция удаленного желудка и нижней трети пищевода осуществлена тонкокишечным трансплантатом. С целью адекватного кровоснабжения петли тощей кишки выполнено аутовенозное аортомезентериальное шунтирование с тощекишечной артерией II порядка. Такие операции должны проводиться только в специализированных онкологических клиниках многопрофильных медицинских учреждений. При этом необходим мультидисциплинарный подход врачей различных специальностей, а также опыт операционный бригады в выполнении подобных операций.

Финансирование. Исследование не имело спонсорской поддержки.

Конфликт интересов. Авторы заявляют об отсутствии конфликта интересов.

Для цитирования: Ищенко Р.В., Епифанцев Е.А., Зотов А.С., Головинский С.В., Грицун В.Ю., Смирнов А.В. Пластика пищевода гиперваскуляризированным тонкокишечным трансплантатом в онкологической практике // Клиническая и экспериментальная хирургия. Журнал имени академика Б.В. Петровского. 2021. Т. 9, № 1. С. 94-98. D0I: https://doi. org/10.33029/2308-1198-2021-9-1-94-98

Статья поступила в редакцию 21.12.2020. Принята в печать 29.01.2021.

Ishchenko R.V. ${ }^{1}$, Epifantsev E.A. ${ }^{2}$, Zotov A.S. ${ }^{2}$, Golovinsky S.V. ${ }^{2}$, Gritsun V.Yu. ${ }^{2}$, Smirnov A.V. ${ }^{2}$

${ }^{1}$ Central Clinical Hospital “Russian Railways-Medicine”, 129128, Moscow, Russian Federation

${ }^{2}$ Federal Scientific and Clinical Center for Specialized Types of Medical Care and Medical Technologies of the FMBA of Russia, 115682, Moscow, Russian Federation

A clinical case of successful surgical treatment of a patient with multiple primary synchronous lung and stomach cancer is presented. Esophageal Reconstruction was performed with an jejunal 
graft. For the purpose of adequate blood supply to the jejunal loop, autovenous aorto-mesenteric shunting with a jejunal artery of the II order was performed. Such operations should be carried out only in specialized oncological clinics of multidisciplinary medical institutions. This requires a multidisciplinary approach involving doctors of various specialties, as well as the experience of the operating team in performing such operations.

Funding. The study had no sponsor support.

Conflict of interests. The authors declare no conflict of interests.

For citation: Ishchenko R.V., Epifantsev E.A., Zotov A.S., Golovinsky S.V., Gritsun V.Yu., Smirnov A.V. Use of supercharged jejunal flap for esophageal reconstruction in oncology practices. Clinical and Experimental Surgery. Petrovsky Journal. 2021; 9 (1): 94-8. DOI: https://doi.org/10.33029/2308-1198-2021-9-1-94-98 (in Russian)

Received 21.12.2020. Accepted 29.01.2021.

\section{Keywords:}

plastic surgery of the esophagus, Jejunal graft, aorto-mesenteric shunting
$P$ еконструктивная пластика пищевода после радикальных оперативных вмешательств - сложная и не до конца решенная задача торакоабдоминальной хирургии. Одним из определяющих факторов успеха всей операции является выбор пластического материала для формирования «неопищевода». Высокие пластические свойства, хорошая васкуляризация, надежность в формировании стебельчатого трансплантата - все это определяет желудок как самый подходящий для пластики орган [1]. Однако использовать желудок можно далеко не во всех ситуациях. В последнее время в литературе все чаще встречаются сообщения о применении в качестве трансплантата в пищеводной хирургии гиперваскуляризированного тонкокишечного кондуита [2].

Приводим собственное клиническое наблюдение, цель которого - показать возможность успешного использования гиперваскуляризированного тонкокишечного трансплантата в качестве пластического материала в реконструктивной хирургии пищевода.

\section{Клиническое наблюдение}

В хирургическое отделение ФГБУ ФНКЦ ФМБА России 08.03.2019 был госпитализирован пациент М., 66 лет, с жалобами на слабость, периодическую гипертермию до $37,5{ }^{\circ} \mathrm{C}$, кашель с отделением слизистой мокроты и периодическими вкраплениями прожилок крови, снижение массы тела на 10 кг за последние 1,5 мес.

Из анамнеза известно, что заболевание манифестировало немотивированной слабостью около 3 мес назад. Постепенно нарастал кашель с отделением слизистой мокроты с прожилками крови. В связи с сохранением жалоб в феврале 2019 г. госпитализирован в стационар по месту жительства.
По данным рентгенографии грудной клетки $(R g):$ затемнение в корне правого легкого до 4 см с перифокальным лимфангиитом.

По данным компьютерной томографии (КT): образование размерами до $9 \times 8$ см в нижней доле правого легкого с инвазией в костальную плевру (рис. 1).

По данным трансторакальной трепан-биопсии верифицирован немелкоклеточный рак правого легкого умеренной степени дифференцировки с массивными очагами некроза.

В ходе онкологического поиска при эзофагогастродуоденоскопии (ЭФГДС) у пациента выявлен рак желудка с мультицентрическим ростом и поражением кардиального и антрального отделов. В дальнейшем пациент был направлен в ФГБУ ФНКЦ ФМБА России. Ситуация обсуждена на онкологическом консилиуме. Рекомендовано проведение комбинированного лечения.

Учитывая наличие первично-множественного синхронного рака правого легкого и желудка, первым этапом решено было выполнить хирургическое вмешательство. 12.03.2019 пациент оперирован.

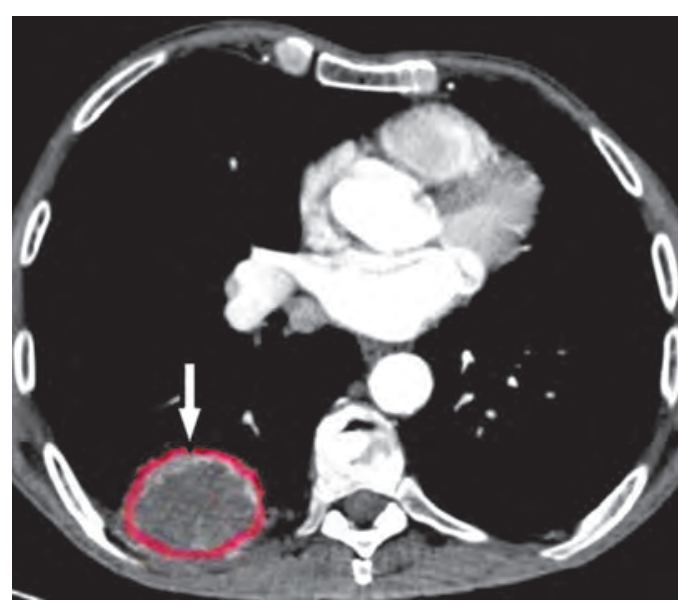

Рис. 1. Компьютерная томограмма. Опухоль нижней доли правого легкого с инвазией в костальную плевру

Fig. 1. CT. Tumor of the lower lobe of the right lung with invasion into the costal pleura 


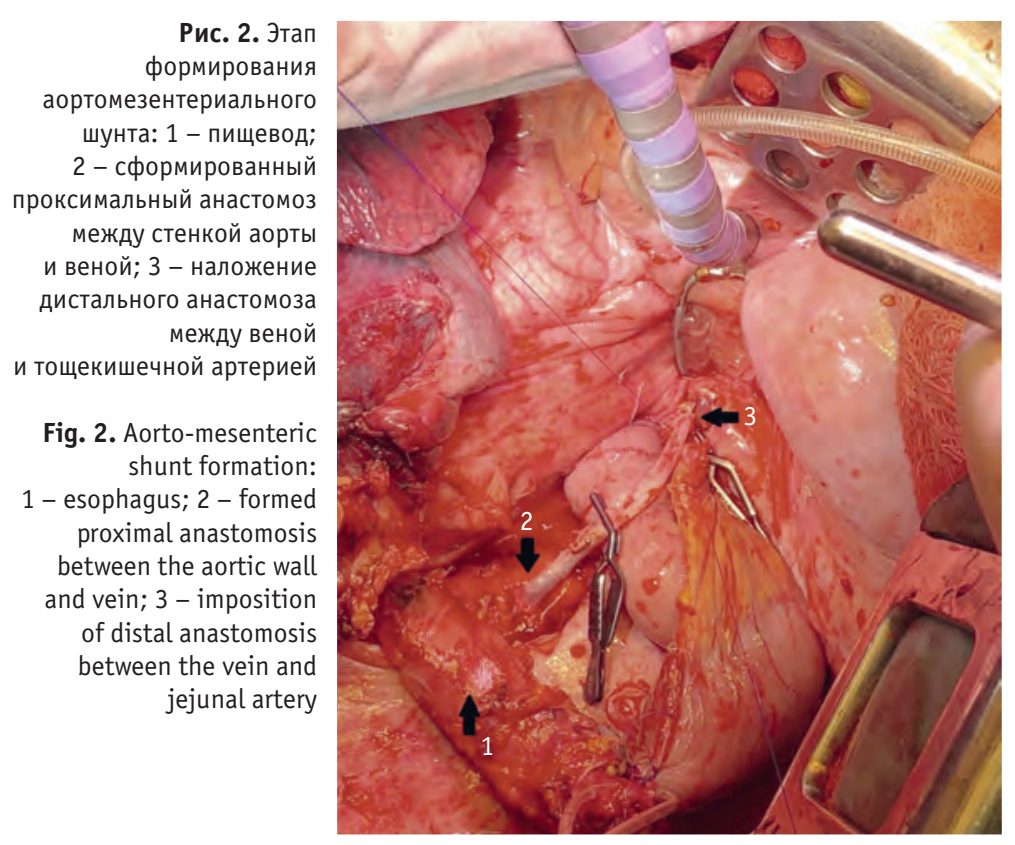

Выполнена верхнесрединная лапаротомия. При интраоперационной ревизии данных за генерализацию процесса не получено. Случай признан операбельным. Выполнена стандартная гастрэктомия с D2-лимфодиссекцией. Препарат отсечен в области абдоминального отдела пищевода, отступя 3 см от Z-линии.

Учитывая данные ЭФГДС и результаты биопсии, край резекции пищевода отправлен на срочное гистологическое исследование, при котором обнаружены атипичные клетки с подозрением на рак. Принято решение о расширении объема оперативного вмешательства до гастрэктомии с резекцией нижнегрудного отдела пищевода и формированием позадиободочного эзофагоеюноанастомоза.

Отступя 30 см от связки Трейтца, сформирована длинная петля тощей кишки по Ру с сохранением питающей артериальной аркады. Отступя от дистальной культи тощей кишки 5 см, наложена микроеюностома для последующего кормления пациента в ближайшем послеоперационном периоде.

Пациент переведен в положение на левый бок. Выполнена правосторонняя переднебоковая торакотомия по шестому межреберью. В плевральной полости - выраженный спаечный процесс в области верхней, средней и нижней долей легкого с париетальной плеврой, произведен адгезиолизис. В нижней доле правого легкого - образование плотной консистенции максимальным размером до 9×8 см с инвазией в париетальную плевру и мягкие ткани грудной стенки. Нижняя доля мобилизована, отделена от грудной стенки. Выполнена расширенная нижнедолевая лобэктомия справа с резекцией IV-VII ребер и грудной стенкой, D2-лимфодиссекцией.
Петля тощей кишки позадиободочно, через диафрагму, выведена в полость заднего средостения для формирования анастомоза с пищеводом. Учитывая натяжение кишки и неудовлетворительный кровоток по прилегающей тощекишечной артерии II порядка, принято решение о выполнении аортомезентериального шунтирования с указанной ветвью (рис. 2). Выполнен забор большой подкожной вены справа от уровня нижней трети до средней трети голени. Грудной отдел аорты выделен на протяжении 6 см на уровне предполагаемого эзофагоеюноанастомоза. Тупым и острым путем обнажена латеральная стенка аорты. Выполнено пристеночное отжатие аорты, выкусывание ее стенки диаметром 4 мм.

Сформирован анастомоз по типу «конец в бок» между грудной аортой и аутовеной непрерывным обвивным швом нитью пролен 6/0. Следующим этапом наложен анастомоз по типу «конец в конец» между аутовеной и тощекишечной артерией II порядка непрерывным обвивным швом нитью пролен 8/0.

Запущен кровоток, определяется отчетливая пульсация венозного шунта и артерии дистальнее сформированного анастомоза. С помощью ультразвуковой допплерографии (УзДГ-флоуметрии) измерен кровоток по шунту: кровоток удовлетворительный (25 мл/мин, сопротивление - 4,0).

Выполнена мобилизация нижнегрудного отдела пищевода с его последующим отсечением с помощью аппарата УД0-40, сформирован эзофагоеюноанастомоз по Бондарю на уровне культи нижней легочной вены (рис. 3).

Таким образом, пациенту удалось выполнить симультанную операцию: 1. Гастрэктомия с резекцией нижнегрудного отдела пищевода с формированием эзофагоеюноанастомоза по Бондарю, аутовенозное аортомезентериальное шунтирование (с тощекишечной артерией II порядка), лимфодиссекция D2, микроеюностомия. 2. Нижняя лобэктомия справа с резекцией грудной стенки и медиастинальной лимфодиссекцией.

Общая кровопотеря во время операции составила 600 мл, в состав операционной бригады входили хирург-онколог, торакальный хирург, кардиохирург, абдоминальный хирург.

По данным планового гистологического исследования выявлена высокодифференцированная аденокарцинома желудка с мультицентрическим ростом (pT1bNOMO) и крупноклеточная карцинома нижней доли правого легкого (рT4NOM0).

Послеоперационный период протекал гладко, больной выписан из стационара на 15-е сутки после операции (из них 3 сут провел в отделении реанимации и интенсивной терапии). При контрольных исследованиях через 1 мес отмечена удовлетворительная проходимость венозного шунта. 


\section{Обсуждение}

В реконструктивно-пластической хирургии пищевода очень важно восстановить непрерывность верхних отделов желудочно-кишечного тракта. Особая сложность возникает при невозможности использовать желудочный трансплантат. В этом случае самый надежный метод для пластики пищевода - использование толстой кишки [3]. К недостаткам пластики пищевода толстой кишкой стоит отнести формирование как минимум 3 анастомозов и относительную техническую сложность оперативного вмешательства.

Если нет возможности использовать толстокишечный трансплантат ввиду различных ситуаций, наиболее приемлемым способом для пластики пищевода остается формирование тонкокишечного кондуита $[4,5]$. К достоинствам тонкой кишки как пластического материала стоит отнести анатомическую схожесть с пищеводом и хорошие функциональные качества трансплантата благодаря сохранению его перистальтической активности.

Однако из-за ряда важных особенностей тонкокишечный кондуит не стал материалом выбора для пластической хирургии пищевода. В первую очередь к таким недостаткам стоит отнести ограниченную протяженность тонкокишечного трансплантата, обусловленную длиной брыжейки кишки, которую невозможно пересечь без риска нарушения кровоснабжения. По мнению многих авторов, данный недостаток может быть нивелирован применением шунтирующих операцией с артериями тонкой кишки с целью гиперваскуляризации [6].

Кроме того, в настоящее время остается дискутабельным вопрос о тактике хирургического лечения пациентов с синхронным раком желудка и раком легкого, что обусловлено редкой встречаемостью данной патологии в структуре онкологических заболеваний [7].

В данном клиническом наблюдении оперативное вмешательство на первом этапе проведено ввиду низкой эффективности химиотерапии на имеющейся стадии заболевания. На основании данных предоперационного обследования с верификацией диагноза случай был признан операбельным, и по решению онкологического консилиума рекомендовано проведение хирургического лечения первым этапом. Учитывая относительную редкость данной патологии, объем оперативного вмешательства, наличие сопутствующей патологии, такие пациенты нуждаются в мультидисциплинарном подходе к планированию и проведению оперативного вмешательства. Определяющим этапом успешного исхода в лечении данной категории пациентов считаем проведение адекватной предоперационной подготовки пациентов, при необходимости с коррекцией белкового обмена и увеличением функциональных резервов легких.

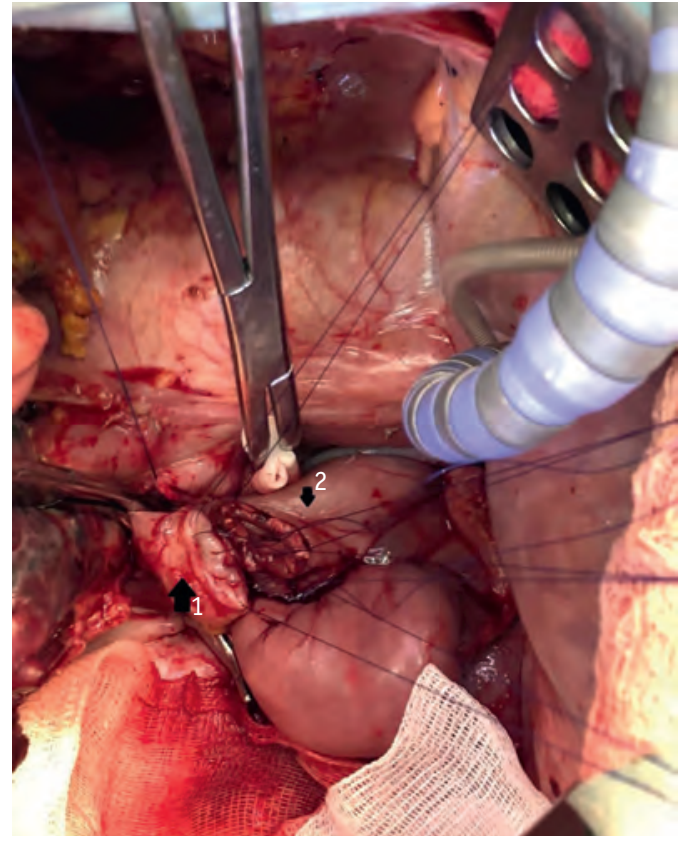

В представленном клиническом случае необходимость использования тонкокишечного кондуита была обусловлена рубцовыми изменениями в брыжейке толстой кишки после ранее проведенных оперативных вмешательств на органах брюшной полости. Такая ситуация стала противопоказанием к выполнению толстокишечной пластики.

Особое место в пластической хирургии пищевода занимает оценка кровоснабжения трансплантата, особенно важно это при формировании «неопищевода» из тонкокишечного трансплантата. Так, в литературе описывается множество вариантов оценки кровоснабжения [2, 3], в нашей клинике мы отдаем предпочтение УЗДГ-флоуметрии как наиболее экономически выгодному и воспроизводимому методу с хорошей чувствительностью. Учитывая возможность междисциплинарного взаимодействия (участие в операции абдоминального, торакального, сосудистого хирургов и онколога) при хирургическом лечении данного пациента удалось достичь благоприятного послеоперационного результата.

\section{Заключение}

Клиническое наблюдение демонстрирует возможность успешного хирургического лечения при конкурирующей опухолевой патологии, а также эффективность использования гиперваскулярного тонкокишечного трансплантата при пластике пищевода. Однако подобное лечение целесообразно и безопасно проводить только в условиях многопрофильных или специализированных онкологических и хирургических стационаров, имеющих в своем составе мультидисциплинарную команду врачей-специалистов и опыт ведения таких пациентов.
Рис. 3. Этап формирования эзофагоеюноанастомоза по Бондарю: 1 - пищевод 2 - тощая кишка

Fig. 3. Formation of esophagojejunoanastomosis according to Bondar: 1 esophagus; 2 - jejunum 


\section{Литература}

1. Adsay N.V., Zamboni G. Paraduodenal pancreatitis: a clinico-pathologically distinct entity unifying "cystic dystrophy of heterotopic pancreas", "para-duodenal wall cyst", and "groove pancreatitis" // Semin. Diagn. Pathol. 2004. Vol. 21, N 4. P. 247-254. DOI: https://doi. org/10.1053/j.semdp.2005.07.005

2. Кригер А.Г., Смирнов А.В., Берелавичус С.В., Горин А.С., Ветшева Н.Н., Нерестюк Я.И. и Ар. Кистоз ная трансформация Авенадцатиперстной кишки при хроническом панкреатите // Исследования и практика в медицине. 2016. T. 3, № 3. C. 49-58. DOI: https://doi. org/10.17709/2409-2231-2016-3-3-5

3. Arvanitakis M., RigauxJ., ToussaintE., Eisendrath P., Bali M.A., Matos C. et al. Endotherapy for paraduodena pancreatitis: a large retrospective case series // Endos copy. 2014. Vol. 46, N 7. P. 580-587. URL: https://doi. org/10.1055/s-0034-1365719

4. Arora A., Rajesh S., Mukund A., PatidarY., ThaparS. Arora A. et al. Clinicoradiological appraisal of "paraduodenal pancreatitis": pancreatitis outside the pancreas! // Indian J. Radiol. Imaging. 2015. Vol. 25, N 3. P. 303-314. DOI: https://doi.org/10.4103/0971-3026. 161467

5. de Pretis N., Capuano F., Amodio A., Pellicciari M., Casetti L., Manfredi R. et al. Clinical and morphological features of paraduodenal pancreatitis: an Italian experience with 120 patients // Pancreas. 2017. Vol. 46, N 4. P. 489-495. DOI: https://doi.org/10.1097/ MPA.0000000000000781

6. Rebours V., Lévy P., Vullierme M.P., Couvelard A., O-Toole D., Aubert A. et al. Clinical and morphologi-

\section{References}

1. Adsay N.V., Zamboni G. Paraduodenal pancreatitis: a clinico-pathologically distinct entity unifying "cystic dystrophy of heterotopic pancreas", "para-duodenal wall cyst", and "groove pancreatitis". Semin Diagn Pathol. 2004; 21 (4): 247-54. DOI: https://doi.org/10.1053/j. semdp.2005.07.005

2. Kriger A.G., Smirnov A.V., Berelavichus S.V. Gorin D.S., Vetsheva N.N., Nerestyuk J.I., et al. Cystic degeneration of the duodenum associated with chronic pancreatitis. Issledovaniya i praktika v medicine [Research and Practice in Medicine]. 2016; 3 (3): 49-58. DOI: https:// doi.org/10.17709/2409-2231-2016-3-3-5 (in Russian)

3. Arvanitakis M., Rigaux J., Toussaint E., Eisendrath P., Bali M.A., Matos C., et al. Endotherapy for paraduodenal pancreatitis: a large retrospective case series. Endoscopy. 2014; 46 (7): 580-7. URL: https://doi. org/10.1055/s-0034-1365719

4. Arora A., Rajesh S., Mukund A., PatidarY., ThaparS., Arora A., et al. Clinicoradiological appraisal of "paraduodenal pancreatitis": pancreatitis outside the pancreas! Indian J Radiol Imaging. 2015; 25 (3): 303-14. DOI: https://doi.org/10.4103/0971-3026.161467

5. de Pretis N., Capuano F., Amodio A., Pellic ciari M., Casetti L., Manfredi R., et al. Clinical and morphological features of paraduodenal pancreati tis: an Italian experience with 120 patients. Pancreas. 2017; 46 (4): 489-95. DOI: https://doi.org/10.1097/ MPA.0000000000000781

6. Rebours V., Lévy P., Vullierme M.P., Couvelard A., O-Toole D., Aubert A., et al. Clinical and morphological cal features of duodenal cystic dystrophy in heterotopic pancreas // Am. J. Gastroenterol. 2007. Vol. 102, N 4. P. 871-879. DOI: https://doi.org/10.1111/j.1572 0241.2007.01091.x

7. Egorov V.I., Vankovich A.N., Petrov R.V., Starostina N.S., Butkevich A.Ts., Sazhin A.V. et al. Pancreaspreserving approach to "paraduodenal pancreatitis" treatment: why, when, and how? Experience of treatment of 62 patients with duodenal dystrophy // Biomed. Res. Int. 2014. Vol. 2014. Article ID 185265. DOI: https://doi. org/10.1155/2014/185265

8. Kempeneers M.A., Issa Y., Ali U.A., Baron R.D., Besselink M.G., Büchler M. et al.; Working Group for the International (IAP - APA - JPS - EPC) Consensus Guidelines for Chronic Pancreatitis. International consensus guidelines for surgery and the timing of intervention in chronic pancreatitis // Pancreatology. 2020 Vol. 20, N 2. P. 149-157. DOI: https://doi.org/10.1016/ j.pan.2019.12.005

9. Yang C.J., Bliss L.A., Freedman S.D., Sheth S., Vollmer C.M., Ng S.C. et al. Surgery for chronic pancreatitis: the role of early surgery in pain management // Pancreas. 2015. Vol. 44, N 5. P. 819-823. DOI: https:// doi.org/10.1097/MPA.0000000000000333

10. Ahmed Ali U., Nieuwenhuijs V.B., van Eijck C.H., Gooszen H.G., van Dam R.M., Busch O.R. et al.; Dutch Pancreatitis Study Group. Clinical outcome in relation to timing of surgery in chronic pancreatitis: a nomo gram to predict pain relief // Arch. Surg. 2012. Vol. 147, N 10. P. 925-932. DOI: https://doi.org/10.1001/archsurg.2012.1094

features of duodenal cystic dystrophy in heterotopic pancreas. Am J Gastroenterol. 2007; 102 (4): 871-9. DOI: https://doi.org/10.1111/j.1572-0241.2007.01091.x

7. Egorov V.I., Vankovich A.N., Petrov R.V., Starostina N.S., Butkevich A.Ts., Sazhin A.V., et al. Pancreaspreserving approach to "paraduodenal pancreatitis" treatment: why, when, and how? Experience of treatment of 62 patients with duodenal dystrophy. Biomed Res Int. 2014; 2014: 185265. DOI: https://doi.org/ $10.1155 / 2014 / 185265$

8. Kempeneers M.A., Issa Y., Ali U.A., Baron R.D., Besselink M.G., Büchler M., et al.; Working Group for the International (IAP - APA - JPS - EPC) Consensus Guidelines for Chronic Pancreatitis. International consensus guidelines for surgery and the timing of intervention in chronic pancreatitis. Pancreatology. 2020; 20 (2): 149-57. DOI: https://doi.org/10.1016/j.pan. 2019.12.005

9. Yang C.J., Bliss L.A., Freedman S.D., Sheth S., Vollmer C.M., Ng S.C., et al. Surgery for chronic pancreatitis: the role of early surgery in pain management. Pancreas. 2015; 44 (5): 819-23. DOI: https://doi.org/10.1097/ MPA.0000000000000333

10. Ahmed Ali U., Nieuwenhuijs V.B., van Eijck C.H., Gooszen H.G., van Dam R.M., Busch O.R., et al.; Dutch Pancreatitis Study Group. Clinical outcome in relation to timing of surgery in chronic pancreatitis: a nomogram to predict pain relief. Arch Surg. 2012; 147 (10): 925-32. DOI: https://doi.org/10.1001/archsurg.2012. 1094 